\title{
L'internationalisation des entreprises : une analyse microéconomique de la mondialisation
}

$\mathbf{L}$ 'accroissement des échanges internationaux de biens, et à un moindre degré de services, est sans aucun doute l'un des bouleversements majeurs qui ont marqué l'économie mondiale au cours des dernières décennies. Le ratio des échanges internationaux de biens et services au PIB mondial, tel qu'enregistré par les balances de paiements, dépasse aujourd'hui nettement les $30 \%$, alors qu'il était inférieur à $13 \%$ au début des années 1970. Encore s'agit-il d'une vision restrictive du phénomène, notamment dans la mesure où la production et la vente sur place des filiales à l'étranger n'est pas comptabilisée en balance des paiements (1). Ainsi, la mondialisation est un phénomène macroéconomique si visible qu'on l'imagine partout, et s'imposant à tous. Or, les données microéconomiques renvoient une image plus nuancée de la réalité. L'analyse de données microéconomiques de commerce international fait ressortir quelques régularités statistiques originales : dans tous les pays du monde, développés ou émergents, la proportion d'entreprises directement engagées dans une relation internationale est très fortement minoritaire. Elle dépasse rarement les $20 \%$. De plus, la plupart des exportateurs n'ont qu'une présence extrêmement limitée sur les marchés mondiaux, en n'étant actifs que sur quelques marchés, voisins de leur pays d'origine (plus de $40 \%$ des exportateurs français, par exemple, ne desservent qu'une seule destination).

Ceci nous rappelle avec force une évidence : même si la mondialisation est un phénomène macroéconomique majeur, même si les économistes et les décideurs politiques ont pris l'habitude d'aborder les questions de compétitivité sous l'angle d'une compétition entre nations, ce ne sont pas les pays qui commercent entre eux, mais bien les entreprises. En s'appuyant sur des outils théoriques nouveaux, et des données individuelles originales, les développements récents de la recherche en économie internationale rétablissent l'équilibre, en laissant plus de place à l'analyse microéconomique de la mondialisation.

\section{Deux théories et demi... plus une}

Krugman avait ouvert un nouveau champ à la théorie du commerce international, avec l'introduction de la concurrence monopolistique, en s'inspirant des travaux de Dixit et Stiglitz (Krugman, 1979). Les échanges étaient fondés sur la demande de variété du consommateur, qui ne pouvait être satisfaite en autarcie en raison des rendements croissants dans la production de chaque variété, rendements croissants découlant eux-mêmes

1. En effet, les exportations et importations, retranscrites dans les balances des paiements, ne concernent que les flux de biens et services qui traversent les frontières. Mais les entreprises peuvent aussi faire le choix de desservir les marchés étrangers en y implantant une filiale de production. 
de la présence de coûts fixes (2). Les différences de coûts comparés liées aux dotations factorielles ou à la technologie n'étaient dès lors plus nécessaires à l'apparition d'un commerce international, même si de telles différences restaient compatibles avec l'existence d'échanges croisés de produits similaires (Helpman et Krugman, 1985). Cette approche a constitué pendant longtemps l'épine dorsale des travaux d'économie internationale appliquée, comme dans le cas du modèle de gravité des échanges (3). Au cœur de cette nouvelle théorie des échanges, on trouve une firme représentative disposant d'un monopole sur la variété (unique) qu'elle produit, mais soumise à la concurrence des autres variétés (des autres firmes), en l'absence de barrières à l'entrée.

Bien entendu, les approches en termes de comportements stratégiques n'étaient pas abandonnées pour autant, notamment sous l'influence de Brander qui a étudié le comportement d'oligopoles internationaux (Brander 1981, Brander et Krugman 1983). Mais ces éléments théoriques ne se prêtant pas aisément à une analyse en équilibre général, ils se sont développés « à l'ombre » de la concurrence monopolistique, sans jamais atteindre le même degré d'influence. Ceci fera dire à Krugman, selon une anecdote rapportée par Neary, que l'on disposait au final de deux théories et demi du commerce international : les deux théories entières étant la concurrence parfaite (et l'avantage comparatif) et la concurrence monopolistique, les analyses oligopolistiques du commerce international ne constituant qu'un ensemble très incomplet (Neary, 2009). Mais surtout, l'approche de Krugman ne saurait rendre compte de deux réalités empiriques largement documentées. D'une part, les échanges croisés de variétés au niveau international portent pour l'essentiel sur des variétés d'un même bien proposées à des prix différents (Fontagné, Freudenberg et Gaulier, 2006). Ce fait stylisé suggère une spécialisation des pays (ou des firmes) sur des niveaux de qualité différents, alors que chez Krugman la différenciation des produits est de nature horizontale. D'autre part, on observe une très forte hétérogénéité des firmes, en termes de productivité, de niveau de salaire moyen, de taille, etc. et seules les plus efficaces accèdent aux marchés internationaux (Mayer et Ottaviano, 2008). Parmi ces dernières, un dernier effet de sélection joue pour déterminer celles s'implantant à l'étranger (Fontagné et Toubal, 2010).

Les travaux les plus récents en économie internationale s'emploient à repousser cette double frontière des connaissances : qualité et hétérogénéité. Pour ce numéro d'Économie et Statistique, nous avons choisi de traiter la seconde de ces dimensions. Le tournant de la littérature est ici, faut-il le rappeler, l'article de Melitz dans Econometrica (Melitz, 2003) suivi d'un second article intégrant le choc concurrentiel de l'ouverture (Melitz et Ottaviano, 2008). Dans ce deuxième article, les marges (i.e. la différence entre le prix proposé et le coût marginal de production) sont endogènes, dans la lignée d'Ottaviano et al. (2002). L'idée d'hétérogénéité des firmes en concurrence monopolistique peut se retrouver ailleurs (Chamberlin, 1933 ; Montagna, 1995 ; Jean, 2002), mais c'est avec la formulation simple et adaptable à différents problèmes proposée par Melitz que la page des modèles de commerce international à firme représentative est vraiment tournée. Ce

2. Les modèles en concurrence monopolistique supposent qu'au sein d'un secteur donné, un grand nombre de firmes assurent la production. Chacune propose une "variété » spécifique du même bien, c'est-à-dire en somme qu'elles proposent leur propre marque. La présence de rendements croissants assure que chaque producteur dispose d'un monopole sur la production de sa propre variété. De leur côté, les consommateurs perçoivent les différences entre ces variétés (elles sont donc imparfaitement substituables) et cherchent à diversifier leur consommation. L'ouverture commerciale permet aux consommateurs d'avoir accès à l'ensemble des variétés produites à l'étranger. Cette demande pour les variétés étrangères donne lieux à des flux simultanés d'importation et d'exportations au sein de chaque catégorie de produits.

3. Le modèle de gravité est très largement utilisé dans les analyses empiriques du commerce international. II prédit que le volume des échanges bilatéraux s'accroit avec la taille économique des deux pays partenaires, et décroit avec la distance géographique et culturelle qui les sépare. Longtemps restées sans fondement théorique explicite, ces équations de gravité ont trouvé une justification explicite avec les théories du commerce en concurrence monopolistique. 
travail a connu deux prolongations immédiates. La première concerne la sélection des firmes s'implantant à l'étranger (Helpman et al., 2004), et précise ainsi les comportements d'entreprises qui doivent faire le choix entre exporter et desservir directement le marché de destination via une filiale (Brainard, 1997), qui constituait depuis une décennie l'explication centrale des investissements directs horizontaux (par réplication des unités de production dans le pays de destination des ventes). La seconde extension concerne la macroéconomie et le cycle des affaires (Ghironi et Melitz, 2005).

La littérature, notamment empirique, s'étant développée sur ces différents thèmes est littéralement pléthorique et il est exclu d'en donner dans cette introduction ne serait-ce qu'une brève revue. Le lecteur trouvera les principales références citées dans les articles du présent numéro. Les économistes français ont largement participé à cette littérature, grâce à la conjonction d'excellentes données à leur disposition et d'une certaine tradition de l'économie internationale appliquée. Les données mobilisées justifiaient qu'un numéro d'Économie et Statistique rende compte de ces travaux : Enquête annuelle d'entreprise, Enquête sur les liaisons financières entre sociétés (LIFI), données individuelles douanières, etc.. C'est donc la qualité du système statistique français d'observation des entreprises, comme la possibilité d'apparier les données, qui a permis de mener à bien ces travaux. Au passage saluons les travaux du Comité du secret statistique du Cnis, soumis à l'examen de multiples demandes émanant du monde académique.

Comme l'illustre ce numéro, cette nouvelle approche en économie internationale donne l'occasion d'apporter un éclairage nouveau non seulement à des questions de théorie économique, mais aussi à des questions de politique économique : effets de la libéralisation commerciale, concurrence Nord-Sud, intégration européenne, contraintes financières, fractionnement des chaînes de valeur, échanges de services, investissement direct étranger.

\section{L'impact de la libéralisation commerciale}

Sitôt que l'on prend en considération le fait que toutes les entreprises n'ont pas les mêmes cartes en main pour profiter de l'ouverture commerciale et y faire face, les conséquences de la mondialisation n'ont rien de trivial. D'un côté, l'abaissement des barrières aux échanges, quelle que soit leur nature, doit permettre un essor des exportations nationales, soit par l'augmentation du nombre d'entreprises exportatrices soit par l'accroissement des ventes de chaque exportateur en place. D'un autre côté, l'ouverture des marchés à la concurrence internationale impose à chacun de réagir.

Ines Buono et Guy Lalanne se penchent sur la première question en étudiant en détail la réaction des exportateurs français aux abaissements de droits de douanes qui ont résulté de la clôture du cycle de négociation du GATT dans les années 1990. L'objectif est de mesurer l'impact de cette libéralisation sur le nombre d'exportateurs (on parle de marge extensive du commerce) et sur les volumes exportés par chaque entreprise (la marge intensive). Ce travail de découpage des variations des volumes de commerce international n'est pas un simple jeu technique. Il conduit à des interprétations importantes pour la politique économique. Même si les données de protection commerciale mobilisées rendent imparfaitement compte du mouvement à l'œuvre, leur travail économétrique suggère que l'essentiel de l'accroissement des exportations françaises lié à la baisse des droits de douane a transité par la marge intensive, ce qui signifie que les exportateurs 
en place sont les principaux bénéficiaires de ces accords commerciaux. Ces entreprises étant globalement plus productives que les autres, leur expansion suggère que la productivité moyenne de l'industrie française a pu croître. Mais cela révèle aussi un problème sérieux. Le manque de réaction de la marge extensive laisse en effet entendre que les petites et moyennes entreprises ont des difficultés (ou des réticences) à profiter de l'ouverture pour se lancer sur les marchés internationaux. Cette conclusion fait donc écho aux travaux (comme ceux présentés dans ce numéro et mentionnés plus loin) qui cherchent à identifier les blocages qui limitent les capacités d'internationalisation des entreprises françaises.

Le second aspect de l'ouverture commerciale - l'intensification de la concurrence internationale - est le thème du travail de Claire Lelarge et Benjamin Nefussi qui examinent la concurrence des pays émergents et étudient comment les firmes françaises montent en gamme pour y échapper. Ceci approfondit le constat fait par Schott (2004) sur données américaines et Fontagné, Gaulier et Zignago (2008) au niveau mondial, dans les deux cas à partir de données agrégeant l'ensemble des firmes d'un pays exportant une même catégorie de produits au cours d'une année. Les effets d'agrégation peuvent être importants et masquer des effets de sélection : il est possible que seules les firmes proposant des variétés de meilleure qualité et pratiquant les prix les plus élevés résistent mieux à cette concurrence du Sud et que la montée en gamme observée ne résulte que d'un effet de composition. D'où l'intérêt de ce travail montrant que les entreprises les plus productives innovent plus en réponse à cette nouvelle concurrence, en ligne avec la thèse de Thoenig et Verdier (2003) relative à l'innovation défensive. S'agissant d'entreprises « diversifiées », cette étude pose la question complexe de la gestion optimale par les firmes multi-produits de leur portefeuille d'activités, thème associé à deux approches distinctes représentées par Bernard et al., (2010) et Eckel et Neary (2010). Ce travail trouve une confirmation et une extension dans la récente analyse de Méjean et Martin (2010), qui souligne une véritable montée en qualité au sein des firmes face à cette concurrence.

C'est toutefois une autre recherche à laquelle fait écho l'article de Julien Martin et Isabelle Méjean dans ce numéro. Suite à l'article controversé de Rose (2000) sur l'impact attendu sur les échanges de l'introduction de l'euro, les estimations ont été revues régulièrement à la baisse. Les données individuelles de firmes exportatrices confirment que l'impact est limité et surtout mettent à jour des effets non triviaux sur le nombre d'exportateurs, le nombre de produits exportés par destination, et la valeur des flux élémentaires (Berthou et Fontagné, 2008). Mais surtout, l'euro renforce les pressions concurrentielles au sein de la zone intégrée, en favorisant les comportements d'arbitrage. La dispersion des prix pratiqués par une même firme sur ses différents marchés, à l'intérieur et à l'extérieur de la zone euro, avant et après l'introduction de la monnaie unique est l'expérience naturelle permettant de répondre à cette question. Au-delà de la réponse, pour le moins nuancée, à la question de départ, l'intérêt de cet article est d'ouvrir la voie à une réflexion sur l'hétérogénéité des firmes non pas en termes de performances, mais de comportement (ici de discrimination par les prix).

\section{Les barrières à l'échange ne sont pas toujours celles qu'on pense}

Comme l'ont montré Buono et Lalanne, peu d'entreprises sont capables de répondre favorablement aux nouvelles opportunités offertes par l'ouverture des marchés mon- 
diaux. Au-delà des protections commerciales formelles, il semble bien que les entreprises font face à un ensemble de barrières qui leur interdisent concrètement d'accéder au statut d'exportateur. Il y a là, à l'évidence, un chantier important pour la politique économique. Notons au passage qu'il ne s'agit pas simplement de chercher à maximiser, dans une démarche mercantiliste, le nombre d'exportateurs et le montant global des exportations, mais bien de repérer les contraintes limitant les capacités d'expansion des firmes de sorte à leur donner les outils nécessaires pour se développer et profiter des opportunités offertes par la mondialisation. Ces contraintes peuvent peser sur la demande (ce sera le cas par exemple en présence de réglementations restrictives sur les marchés d'exportation), mais aussi sur l'offre, en particulier dès lors qu'il s'agit de financer l'accès au statut d'exportateur.

On s'attend ainsi à ce que les exportateurs contraints financièrement aient un accès plus difficile aux marchés étrangers, comme le suggère le résultat de Manova (2008) selon lequel la libéralisation financière a un impact positif plus marqué sur les secteurs dépendants de la finance externe (Rajan et Zingales, 1998). Ce type de question a trouvé un écho renouvelé à l'occasion de la forte chute du commerce international entre le dernier trimestre de l'année 2008 et le premier trimestre 2009. Amiti et Weinstein (2009) illustrent dans le cas japonais comment le nécessaire recours à la finance externe en matière de commerce international a affecté les exportations des firmes japonaises dans la crise bancaire de 1997. L'importance de cet effet dans le cas de la crise actuelle est pourtant discuté, la demande jouant un rôle important (Levchenko et al., 2010) même si les firmes les plus contraintes financièrement ont vu leurs performances exportatrices se dégrader plus fortement (Bricongne et al., 2010). L'intérêt de l'article de Flora Bellone, Patrick Musso, Lionel Nesta et Stefano Schiavo, outre l'emploi de données individuelles, est finalement de distinguer entre impact de la santé financière sur la décision d'exporter et impact de l'exportation sur la santé financière.

Mais les blocages ne sont pas uniquement d'ordre financier, ni même purement économiques. Ils peuvent aussi être plus diffus et plus informels. Commencer à exporter, ou simplement se positionner sur un nouveau marché étranger, n'est pas un acte anodin. Les entreprises qui exportent, même en direction de pays géographiquement et culturellement proches, doivent faire face à un environnement nouveau, différent de celui dans lequel elles ont l'habitude d'évoluer. Outre les difficultés techniques (les formalités douanières et logistiques), les entreprises doivent acquérir des informations spécifiques sur chaque marché étranger, y trouver des partenaires et des clients, rédiger des contrats et découvrir le climat social et juridique dans lequel elles devront travailler. Identifier et quantifier ces difficultés à l'aide d'outils d'analyse empirique systématique n'est pas chose facile. Une façon de montrer leur existence consiste à quantifier les externalités d'exportations. Koenig (2009) a montré qu'une entreprise avait d'autant plus de chance de commencer à exporter vers un pays qu'il y a, dans son environnement géographique proche, d'autres exportateurs vers ce même pays. Pamina Koenig, Florian Mayneris et Sandra Poncet vont plus loin et montrent que ces externalités comptent d'autant plus que le marché étranger a des institutions fragiles.

\section{La mondialisation, c'est aussi les services}

Souvent considérés comme nonéchangeables, les secteurs de services ne sont pourtant pas exclus de la mondialisation. Les échanges de services enregistrés dans les balances 
des paiements représentent environ $20 \%$ du commerce mondial. C'est peu au regard du poids des services dans les économies développés, mais ce chiffre est appelé à croître dans les prochaines années au fur et à mesure de l'avancée des négociations internationales sur la libéralisation des échanges de services. Rappelons de surcroît qu'une fraction non négligeable des ventes de services à l'étranger passe par la présence sur place, et donc échappe aux statistiques de balances des paiements. Même si l'on se limite aux échanges des services qui se font explicitement entre résidents nationaux et non-résidents, le manque de données sectorielles détaillées sur ce type de commerce, du moins en comparaison de l'extraordinaire foisonnement des bases d'informations sur les échanges de biens, a limité le nombre d'analyses précises des déterminants du commerce de services. Mais là où les matrices de données sectorielles et multi-pays font défaut, les bases d'information sur les entreprises nous éclairent sur la profondeur de la mondialisation dans les différentes activités de services. C'est à cela que s'attachent deux contributions présentées dans ce numéro : celle de Muriel Barlet, Laure Crusson, Sébastien Dupuch et Florence Puech et celle de Guillaume Gaulier, Emmanuel Milet et Daniel Mirza.

Les premiers tentent de mesurer le degré « d'échangeabilité » des différents secteurs de services. En effet, il ne suffit pas de constater que seule une petite fraction des secteurs de services fait l'objet d'un échange international pour conclure que tous les autres services sont nécessairement voués à n'exister que sur des marchés locaux. Si certains services ne sont pas échangés à l'international, ce n'est pas pour autant qu'ils sont intrinsèquement inéchangeables; ces secteurs peuvent aussi faire face à des barrières qui interdisent aux entreprises de se positionner sur les marchés étrangers. La méthode utilisée est originale et le résultat surprenant et riche d'enseignement. L'idée de départ est simple : une catégorie de services doit être considérée comme « échangeable », dès lors qu'elle fait l'objet d'échanges à distance à l'intérieur des pays. Les auteurs comparent donc la répartition spatiale des offres de chaque type de services au sein du territoire français à la répartition de la demande : alors que seulement la moitié des catégories de services sont échangées à l'international, plus de $80 \%$ d'entre elles apparaissent comme « échangeables » sur la base du critère ainsi retenu.

Ce résultat rappelle avec force à quel point la mondialisation reste incomplète dans le domaine des services, même si une partie des services, ainsi rendus à distance à l'intérieur d'un pays, peuvent l'être par des filiales de groupes étrangers. Il permet aussi de comprendre pourquoi le sujet fait l'objet de débats si âpres lors des négociations commerciales, et souligne que l'ouverture à la concurrence internationale de ces secteurs est sans aucun doute l'un des défis essentiels auquel doivent se préparer les entreprises. Or, les entreprises de services, même dans les secteurs ouverts, semblent pour l'heure avoir bien des difficultés à exporter. C'est ce que documente le travail de Guillaume Gaulier, Emmanuel Milet et Daniel Mirza. Ceux-ci exploitent une base de données de la Banque de France qui retrace, à l'instar des bases individuelles des douanes, l'ensemble des flux d'exportations de services, pour chaque entreprise française, enregistrés en balance des paiements. Il apparaît que si exporter n'est pas chose facile pour les entreprises industrielles, c'est encore plus compliqué dans le domaine des services. Le nombre d'entreprises françaises exportant des services est extrêmement faible (on en compte dix fois moins que les exportateurs de biens) et les exportations sont très fortement concentrées sur quelques grandes entreprises. 


\section{Les autres formes d'internationalisation}

L'internationalisation des entreprises ne s'arrête pas au seul fait d'exporter. La mondialisation ouvre aussi la possibilité - on l'a déjà souligné - d'accéder directement aux marchés étrangers afin de mieux servir les demandes locales. Mais elle permet aussi de profiter des avantages comparatifs des différents pays pour accroître sa compétitivité en important des biens intermédiaires ou des biens d'équipement. Là encore, les stratégies d'internationalisation des entreprises dépendent de leur capacité à faire face aux défis de la mondialisation, et l'éventail des opportunités offertes par l'ouverture des économies ne sont à la portée que d'un petit nombre.

Alexandre Gazaniol, Frédéric Peltrault et Jean-Marc Siroen soumettent la thèse de Helpman, Mélitz et Yeaple déjà citée à l'épreuve des faits. Ils montrent que les primes à l'implantation de filiales internationales, c'est-à-dire la sur-performance ex ante des firmes présentes à l'étranger, existent et dépassent celles d'exportation. Dit autrement, les entreprises doivent afficher une productivité très élevée pour être en mesure d'exporter une part de leur production, mais, pour être capable d'investir à l'étranger, elles doivent faire preuve de performances exceptionnelles. Fabrice Defever et Farid Toubal vont plus loin dans l'exploration des stratégies d'internationalisation. Ils étudient quels sont les facteurs qui vont guider les entreprises dans leurs décisions de sous-traitance à l'étranger, et notamment leur choix entre créer une filiale internationale ou faire appel à un fournisseur étranger indépendant. Conformément aux prédictions théoriques (avancées notamment par Antràs et Helpman, 2004), ils montrent que le mode d'internationalisation choisi par les entreprises dépend fortement de leur productivité : seules les plus productives préfèreront externaliser la production. Ce résultat met en relief les difficultés à mettre en œuvre des contrats internationaux et souligne, une fois encore, à quel point la mondialisation est, pour les entreprises, un chemin semé d'embûches.

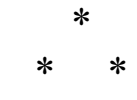

La recherche en économie internationale s'est longtemps cantonnée à des études macroéconomiques ou sectorielles. Quand elle a utilisé des données de commerce très fines au niveau des produits, ces données agrégeaient toujours les différentes firmes exportatrices ou importatrices au niveau national. En s'écartant des modèles de commerce international avec firme représentative, les développements théoriques réalisés depuis le début des années 2000 ouvrent la voie à une approche plus microéconomique des analyses du commerce international. Sur cette base, l'exploitation de données individuelles de commerce et d'investissement international trouve tout son sens. Ces analyses microéconomiques du commerce international offrent un regard nouveau sur la mondialisation, ne serait-ce qu'en montrant que, même dans un pays aussi ouvert aux échanges que la France, exporter n'est en rien facile et banal, si bien que le commerce des grands pays est le fait, pour l'essentiel, de quelques grandes entreprises qui ont su surmonter les difficultés de l'internationalisation.

Surtout, ces travaux ouvrent de nouvelles pistes pour la conduite des politiques économiques. En précisant les conséquences de l'ouverture commerciale pour chaque entreprise, en identifiant les difficultés spécifiques qui freinent l'internationalisation des firmes ou en observant la nature de leurs choix stratégiques, ces travaux permettent de mieux rendre compte des implications des politiques commerciales et de leur limites. Ainsi, 
face aux déséquilibres actuels de la balance commerciale, la France, comme beaucoup d'autres pays, propose des réponses qui relèvent bien plus de la micro que de la macroéconomie. En témoigne la succession de mesures visant à accompagner les entreprises (et notamment les PME) à l'étranger (4), et les politiques de pôles de compétitivité qui affichent la volonté de donner aux entreprises les moyens de faire face à la concurrence étrangère et d'accéder aux marchés mondiaux. Toutefois, et même si certains travaux ont déjà commencé (par exemple, Fontagné et al., 2010), une évaluation quantitative systématique de ces politiques reste à faire.

L'autre voie de recherche évidente qui s'ouvre à nous est, bien sûr, l'exploration des conséquences sociales de la mondialisation. L'ouverture commerciale - rares sont ceux qui en doutent - fait des gagnants mais aussi des perdants. Il est malheureusement fort à craindre que les travailleurs les moins qualifiés fassent partie du lot des perdants. Les études menées sur cette question dans les années 1990 ne sont pas parvenues à des conclusions définitives concernant la responsabilité de l'ouverture aux échanges internationaux dans l'accroissement des inégalités sociales dans les pays développés. À la fin des années 1990, une meilleure prise en compte des stratégies d'internationalisation (et notamment le recours à des sous-traitants pour la réalisation de certains segments de chaîne de valeur) a conduit à relativiser les conclusions initiales en montrant à quel point les moins qualifiés pouvaient être affectés par les conséquences de la concurrence internationale (Feenstra et Hanson, 1999). Nous avons aujourd'hui les outils nécessaires pour aborder cette question avec une approche clairement microéconomique. En effet, les développements théoriques récents proposent des modèles de commerce incorporant explicitement les imperfections du marché du travail (Helpman et al., 2010) et mettent en avant une relation subtile entre ouverture et inégalités. La communauté scientifique dispose aussi de données permettant d'observer l'évolution de l'emploi et les pratiques salariales des entreprises confrontées à la concurrence étrangère. Face à la méfiance qui s'exprime dans beaucoup de pays vis-à-vis de la mondialisation, et afin de pouvoir apporter les réponses politiques nécessaires à la correction de ses conséquences négatives, ces recherches (d'ores et déjà initiées par Biscourp et Kramarz, 2007) demandent à être multipliées et approfondies.

Matthieu Crozet (PSE - Université Paris-I et CEPII) *, Lionel Fontagné (PSE - Université Paris-I et CEPII) **

4. Le site gouvernemental du commerce extérieur pour un panorama complet des mesures existantes (http ://www.exporter.gouv.fr/ exporter/).

* Université Paris I, Maison des Sciences Économiques, 106 boulevard de l'hôpital, 75013 Paris. crozet@univ-paris1.fr.

** Université Paris I, Maison des Sciences Économiques, 106 boulevard de l'hôpital, 75013 Paris. lionel.fontagne@univ-paris1.fr. 


\section{BIBLIOGRAPHIE}

Amiti M. et Weinstein D. (2009), « Exports and Financial Shocks », NBER Working Paper, n 15556.

Antràs P. et Helpman E. (2004), « Global Sourcing », Journal of Political Economy, University of Chicago Press, vol. 112, n 3, pp. 552-580.

Brainard L. (1997), « An Empirical Assessment of the Proximity-Concentration Trade-off Between Multinational Sales and Trade », American Economic Review, vol. 87, n 4, pp. 520-544.

Brander J. (1981), « Intra-Industry Trade in Identical Commodities », Journal of International Economics, vol. $11, \mathrm{n}^{\circ} 1$, pp. 1-14.

Brander J. et Krugman P. (1983), « A 'Reciprocal Dumping' Model of International Trade », Journal of International Economics, vol. 15, $\mathrm{n}^{\circ} 3-4$, pp. 313-321.

Bernard A., Redding S. et Schott P. (2010), « Multiproduct Firms and Trade Liberalization », Quarterly Journal of Economics, à paraître.

Berthou A. et Fontagné L. (2008), « The Euro Effects on the Firm and Product-Level Trade Margins : Evidence from France », Document de travail, Cepii, $\mathrm{n}^{\circ}$ 2008-21.

Biscourp P. et Kramarz F. (2007), « Employment, Skill Structure, and International Trade », Journal of International Economics, vol. 72, $\mathrm{n}^{\circ} 1$, pp. 22-51.

Bricongne J.-C., Fontagné L., Gaulier G., Taglioni D. et Vicard V. (2010), « Firms and the Global Crisis : French Exports in the Turmoil », European Central Bank Working Paper, $\mathrm{n}^{\circ} 1245$.

Chamberlin E. H. (1933), Theory of Monopolistic Competition, Harvard University Press, Cambridge MA.

Eckel C. et Neary P. (2010), « Multi-Product Firms and Flexible Manufacturing in the Global Economy », Review of Economic Studies, vol. 77, n 1, pp. 188-217.

Feenstra R. et Hanson G. (1999), « The Impact of Outsourcing and High-Technology Capital on Wages : Estimates for the U.S., 1972-1990 », Quarterly Journal of Economics, vol. 114, n 3, pp. 907-940.

Fontagné L. et Toubal F. (2010), Investissements directs à l'étranger et performances des entreprises, Rapport du CAE, $n^{\circ} 89$.

Fontagné L., Freudenberg M. et Gaulier G. (2006), « A Systematic Decomposition of World Trade into Horizontal and Vertical IIT», Review of World Economics, vol. 142, n³ 3, pp. 459-475.

Fontagné L., Gaulier G. et Zignago S. (2008), « Specialization Across Varieties and North-South Competition », Economic Policy, vol. 23, n 53, pp. 51-91.

Fontagné L., Koenig P., Mayneris F. et Poncet S. (2010), « Clustering the Winners : the French Policy of Competitiveness Clusters », Document de travail, Cepii, ${ }^{\circ} 2010-18$.

Ghironi F. et Melitz M. (2005), « International Trade and Macroeconomic Dynamics with Heterogeneous Firms », Quarterly Journal of Economics, vol. 120, n³ 3, pp. 865-915.

Helpman E. et Krugman P. (1985), Market Structure and Foreign Trade, MIT Press, Cambridge MA.

Helpman E., Itskhoki O. et Redding S. (2010), « Inequality and Unemployment in a Global Economy », Econometrica, vol. 78, $\mathrm{n}^{\circ}$ 4, pp. 1239-1283.

Helpman E., Melitz M. et Yeaple S. (2004), « Export Versus FDI with Heterogeneous Firms », American Economic Review, vol. 94, n 1, pp. 300-316. 
Jean S. (2002), « International Trade and Firms’ Heterogeneity Under Monopolistic Competition », Open Economies Review, vol. 13, n 3, pp. 291-311.

Koenig P. (2009), "Agglomeration and the Export Decision of French Firms », Journal of Urban Economics, vol. 66, n 3, pp. 186-195.

Krugman P. (1979), « Increasing Returns, Monopolistic Competition and International Trade », Journal of International Economics, vol. 9, n 4, pp. 469-479.

Levchenko A., Lewis A.L. et Tesar L. (2010), « The Collapse of International Trade During the 20082009 Crisis : In Search of the Smoking Gun », IMF Economic Review, vol. 58, n² 2, pp. 214-253.

Manova K. (2008), « Credit Constraints, Equity Market Liberalizations and International Trade », Journal of International Economics, vol. 76, n 1, pp. 33-47.

Mayer T. et Ottaviano G. (2007), The Happy Few : New Facts on the Internationalisation of European Firms. New Facts Based on Firm-Level Evidence, Bruegel-CEPR EFIM 2007 Report, Bruegel Blueprint Series.

Méjean I. et Martin J. (2010), « Low-Wage Countries’ Competition and Quality Upgrading : Firm-Level Evidence », mimeo, Paris School of Economics.

Melitz M. (2003), « The Impact of Trade on Intra-Industry Reallocations and Aggregate Industry Productivity », Econometrica, vol. 71, n 6, pp. 1695-1725.

Melitz M. et Ottaviano G. (2008), « Market Size, Trade, and Productivity », Review of Economic Studies, vol. $75, \mathrm{n}^{\circ} 1$, pp. 295-316.

Montagna C. (1995), « Monopolistic Competition with Firm-Specific Costs », Oxford Economic Papers, vol. $47, \mathrm{n}^{\circ} 2$, pp. 318-328.

Ottaviano G., Tabuchi T. et Thisse J.-F. (2002), «Agglomeration and Trade Revisited », International Economic Review, vol. 43, n 2, pp. 409-436.

Rajan R. et Zingales L. (1998), « Financial Dependence and Growth », American Economic Review, vol. $88, \mathrm{n}^{\circ} 3$, pp. 559-586.

Rose A. (2000), " One Money, One Market : Estimating the Effect of Common Currencies on Trade », Economic Policy, vol. 15, n 30, pp. 7-46.

Schott P. (2004), "Across-Product versus Within-Product Specialization in International Trade », Quarterly Journal of Economics, vol. 119, n² 2, pp. 647-678.

Thoenig M. et Verdier T. (2003), « Theory of Defensive Skill-Biased Innovation and Globalization », American Economic Review, vol. 93, n³, pp. 709-728. 